\title{
Abkürzungen
}

\begin{tabular}{|c|c|}
\hline ABl. & Amtsblatt \\
\hline AELF & Ausschuß für Ernährung, Landwirtschaft und Forsten \\
\hline AfA & Arbeitsgemeinschaft für Agrarfragen \\
\hline ApA & Agrarpolitischer Ausschuß der SPD beim Parteivorstand \\
\hline AsD & Archiv der sozialen Demokratie \\
\hline ASG & Agrarsoziale Gesellschaft \\
\hline BA & Bundesarchiv \\
\hline $\mathrm{BICO}$ & Bipartite Control Office (Frankfurt) \\
\hline BMELF & Bundesminister(ium) für Ernährung, Landwirtschaft und Forsten \\
\hline BMR & Britische Militärregierung \\
\hline BRG & Bodenreformgesetz \\
\hline DBK & Deutsche Bauern-Korrespondenz \\
\hline DBT & Deutscher Bundestag \\
\hline DBV & Deutscher Bauernverband \\
\hline DGB & Deutscher Gewerkschaftsbund \\
\hline DP & Deutsche Partei \\
\hline Drs. & Drucksache \\
\hline DVO & Durchführungsverordnung \\
\hline GF & Gesamtfläche (eines Betriebes) \\
\hline GFK & Gesellschaft zur Förderung der inneren Kolonisation \\
\hline G.S.B. & $\begin{array}{l}\text { Gesetz zur Beschaffung von Siedlungsland und zur Bodenreform } \\
\text { (US-Zone) }\end{array}$ \\
\hline GVOBl. & Gesetz- und Verordnungsblatt \\
\hline HLT & Hannoverscher Landtag \\
\hline IfZ & Institut für Zeitgeschichte \\
\hline JCS & Joint Chiefs of Staff \\
\hline $\mathrm{KdM}$ & Konferenz der Ministerpräsidenten der britischen Zone \\
\hline LK & Landwirtschaftskammer Hannover \\
\hline LNF & Landwirtschaftliche Nutzfläche \\
\hline $\mathrm{LT}$ & Landtag \\
\hline MP & Ministerpräsident \\
\hline MR & Militärregierung \\
\hline Nds. & Niedersachsen \\
\hline NGVOBl. & Niedersächsisches Gesetz- und Verordnungsblatt \\
\hline NL & Nachlaß \\
\hline NLP & Niedersächsische Landespartei \\
\hline NLT & Niedersächsischer Landtag \\
\hline NLV & Niedersächsisches Landvolk \\
\hline NMELF & $\begin{array}{l}\text { Niedersächsisches Ministerium für Ernährung, Landwirtschaft } \\
\text { und Forsten (Minister für ...) }\end{array}$ \\
\hline NRW & Nordrhein-Westfalen \\
\hline NSA & Niedersächsisches Staatsarchiv Hannover \\
\hline NSK & Niedersächsische Staatskanzlei \\
\hline NSM & Niedersächsisches Staatsministerium \\
\hline OMGUS & Office of Military Government for Germany, US-Zone of Occup \\
\hline
\end{tabular}




$\begin{array}{ll}\text { R.C. } & \text { Regional Commissioner } \\ \text { RNS } & \text { Reichsnährstand } \\ \text { RSG } & \text { Reichssiedlungsgesetz } \\ \text { SAfAR } & \text { Sonderausschuß für Agrarreform } \\ \text { SG } & \text { Siedlungsgesetz } \\ \text { SOPADE } & \text { Sozialdemokratische Partei Deutschlands (Parteipublikation) } \\ \text { TO } & \text { Tagesordnung } \\ \text { VELF } & \text { Verwaltung für Ernährung, Landwirtschaft und Forsten im Vereinigten } \\ & \text { Wirtschaftsgebiet (Frankfurt) } \\ \text { VfZG } & \text { Vierteljahrshefte für Zeitgeschichte } \\ \text { VO } & \text { Verordnung } \\ \text { VWG } & \text { Vereinigtes Wirtschaftsgebiet } \\ \text { WAV } & \text { Wirtschaftliche Aufbauvereinigung } \\ \text { WiGBl. } & \text { Gesetzblatt des Wirtschaftsrates des VWG } \\ \text { WR } & \text { Wirtschaftsrat des VWG } \\ \text { ZBR } & \text { Zonenbeirat } \\ \text { ZEL } & \text { Zentralamt für Ernährung und Landwirtschaft in der britischen Zone } \\ & \text { (Hamburg) } \\ \text { ZZVELF } & \text { Zweizonenverwaltung für Ernährung, Landwirtschaft und Forsten } \\ & \text { (Stuttgart) }\end{array}$




\section{Quellen und Literatur}

\section{Unveröffentlichte Quellen}

\section{Bundesarchiv Koblenz}

Akten des ZEL und der VELF -

Büro des Direktors:

\section{$\mathrm{Z} 6 \mathrm{I} / 160-175$}

$\mathrm{Z} 6$ III/48-54

Akten des Zonenbeirats:

$\mathrm{Z} 2 / 5$

Akten des BMELF:

ZA, B 116/1228, 1229, 1231; Az. 4741

ZA, B 116/1170, 1171, 1191, 1211;

$$
\text { Az. } 4703,4720,4730
$$

Nachlaß Schlange-Schönigen:

Bd. 1, 17-20

2. Niedersächsisches Staatsarchiv Hannover (Hauptstaatsarchiv Hannover)

Akten des NMELF:

Nds. Z 600

Acc. 11/63, vol. 1-3

Acc. $45 / 55$, vol. 1-6

Akten der NSK:

Nds. Z 50,

Acc. 32/63, Nr. 27, 31, 46-49, 63, $64,75,77,80,87,92,93,99,107$

Akten der DP:

3. Niedersächsisches Ministerium für Ernährung, Landwirtschaft und Forsten,

Hannover

III A 450 (Bodenreform), 7 Bde.

IV A 450 (Bodenreform-Planung)

- 1/1 Allgemeines, 5 Bde.

- 1/2 Planung (BZ)

- 1/3 Planung (US-Zone)

IV A 450 Planung, - 2/1 - 3

IV A 450 Entwurf Bodenreformgesetz

4. Deutscher Bundestag, Parlamentsarchiv, Bonn

WR des VWG: Sonderkomitee für Bau- und Bodenrecht, Bd.1

WR des VWG: Ausschuß für Ernährung, Landwirtschaft und Forsten, 1947-1949

Zonenbeirat: Agrarausschuß (Ausschuß für Bodenreform) 1946

Sonderausschuß für Agrarreform 1947

5. Archiv der sozialen Demokratie, Bonn-Bad Godesberg

Akten des SPD-Bezirks Braunschweig: Agrarpolitischer Ausschuß (1946-1954)

6. Archiv der CDU-Bundesgeschäftsstelle, Bonn

Altten des Alten Archivs: A 7 b Bodenreform

$$
\begin{aligned}
& \text { A } 7 \text { a, Teil I-IV } \\
& \text { B } 52 \text { g Nds., j, h }
\end{aligned}
$$

7. Agrarsoziale Gesellschaft, Göttingen

D $5 \mathrm{~b} / 1: 1,1: 2,1: 3,1: 4,3$

V 7-9, 13, 14, 19, 22, 110

8. Landesverband des Niedersächsischen Landvolkes, Hannover

1947/48 (I 2 a/2 b), 1949, 1950 


\section{Landwirtschaftskammer Hannover}

Akten zur Vorläufigen Landwirtschaftskammer (Heft 1)

Akten zur Bodenreform $\mathrm{F} 4$

10. Zeugenbefragungen durch den Verfasser

Haxsen, Ottomar, Mitglied des Niedersächsischen Landtags (erst DP, später CDU), seit 1951 Vorsitzender des NLT-Ausschusses für Ernährung, Landwirtschaft und Forsten, Befragung am 5. Februar 1969

Kriedemann, Herbert, Mitglied des Bundestags (SPD), Befragung am 2. Oktober 1970 und am 31. März 1971

Schmalz, Hellmuth, Vorsitzender der Gewerkschaft Gartenbau, Land- und Forstwirtschaft, 1951 von der KPD zur SPD übergetreten, Befragung am 21. Januar 1971

Siemer, Hermann-Josef, Mitglied des Bundestags (CDU), Befragung am 31. März 1971

\section{Veröffentlichte Quellen und Literatur}

Adenauer, Konrad, Erinnerungen, Bd. I, Stuttgart 1965

Agartz, Viktor, Sozialistische Wirtschaftspolitik, Rede auf dem Parteitag der SPD am 9.5.1946 in Hannover, Hamburg 1947

Agrar- und Bodenreform in Schleswig-Holstein, Kiel 1946

Amtsblatt der Militärregierung - Deutschland - (Britisches Kontrollgebiet), Berlin 1945-1949

Amtsblatt für Niedersachsen, Hannover 1947/48

Balabkins, Nicholas, Germany under direct controls. Economic aspects of industrial disarmament 194.5-1948, New Brunswick 1964.

Billerbeck, Rudolf, Die Abgeordneten der ersten Landtage (1946-1951) und der Nationalsozialismus, Düsseldorf 1971

Blum, John M., Deutschland - ein Ackerland, Düsseldorf 1968

Die Bodenreform im Spiegel der Presse, hrsg. von der Pressestelle der Landwirtschaftskammer Hannover, Hannover 1947

Boyens, Wilhelm Friedrich, Siedlung und Bodenreform als Aufgabe des Bundes. Schleswig-Holsteins Beitrag zur Frage der ländlichen Siedlung, Hamburg 1950

Bürger, Hans, Die landwirtschaftliche Interessenvertretung in der Zeit von 1933 bis zur Gegenwart unter besonderer Berücksichtigung der westdeutschen Verhältnisse, Erlangen 1967 (Diss.)

Clay, Lucius D., Entscheidung in Deutschland, Frankfurt 1950

Determinanten der westdeutschen Restauration 1945-1949, Autorenkollektiv: ErnstUlrich Huster u. a., Frankfurt 1972

Deuerlein, Ernst, Deklamation oder Ersatzfriede? Die Konferenz von Potsdam 1945, Stuttgart 1970

ders., CDU/CSU 1945-1954, Köln 1960

ders., Forschungsgrundlage und Forschungsproblematik 1945-1949, in: Politische Studien 22 (1971), S. 46-67

Deutsche Bauern-Korrespondenz, Mitteilungsblatt der Arbeitsgemeinschaft der deutschen Bauernverbände, Bonn 1948/1949

Dorendorf, Annelies, Der Zonenbeirat der britisch besetzten Zone. Ein Rückblick auf seine Tätigkeit, Göttingen 1953

Ebsworth, Raymond, Restoring Democracy in Germany. The British Contribution, London 1960 
Ehrenforth, Werner, Die Bodenreform im Bundesgebiet, in: Deutsche Rechts-Zeitschrift 4. (1949), S. $483 \mathrm{ff}$.

Flechtheim, Ossip K. (Hrsg.), Dokumente zur parteipolitischen Entwicklung in Deutschland seit 1945, Bd. I-III, Berlin $1962 \mathrm{ff}$.

Foerst, Walter, Geschichte Nordrhein-Westfalens, Bd. 1, Köln/Berlin 1970

Freies Niedersachsen [Organ der NLP, später: Niedersächsische Stimmen], Hannover $1946 / 1947$

Friedmann, Wolfgang, The Allied Military Government of Germany, London 1947

Gereke, Günther, Ich war königlich-preußischer Landrat, Berlin 1970

Gimbel, John, The American Occupation of Germany. Politics and the Military 1945-1949, Stanford 1968

ders., Byrnes' Stuttgarter Rede und die amerikanische Nachkriegspolitik in Deutschland, in: VfZG 20 (1972), S. 39-62

ders., Die Konferenzen der deutschen Ministerpräsidenten 1945-1949, in : aus politik und zeitgeschichte B 31/1971, S. 3-28

Girndt, Ilse, Zentralismus in der britischen Zone. Entwicklungen und Bestrebungen beim Wiederaufbau der staatlichen Verwaltungsorganisation auf der Ebene oberhalb der Länder 1945-1948, Bonn 1971 (Diss.)

Häbich, Theodor, Das Recht der Landlosen in der US-Zone, Frankfurt 1947

Hannoverscher Landtag, Stenographische Berichte der 1.-3. Sitzung, Hannover 1946

Hartwich, Hans-Hermann, Sozialstaatspostulat und gesellschaftlicher status quo, Köln 1970

Haushofer, Heinz, Ideengeschichte der Agrarwirtschaft und Agrarpolitik im deutschen Sprachgebiet, Bd. II, München 1958

Heinke, Alfred, Das Potsdamer Abkommen und die Durchführung der Bodenreform in Deutschland, in: Biologie in der Schule 8 (1959), S. 67-71

Herlemann, Hans-Heinrich, Vertriebene Bauern im Strukturwandel der Landwirtschaft, in: Die Vertriebenen in Westdeutschland. Hrsg. von Eugen Lemberg, Friedrich Edding, Bd. 2, Kiel 1959

Hermes, Peter, Die CDU und die Bodenreform in der Sowjetischen Besatzungszone Deutschlands im Jahre 1945, Saarbrücken 1963

Hüttenberger, Peter, Die Anfänge der Gesellschaftspolitik in der Britischen Zone, in: VfZG 21 (1973), S. 171-176

ders., Nordrhein-Westfalen und die Entstehung seiner parlamentarischen Demokratie, Siegburg 1973

Hummel, Johannes, Zur Geschichte des Deutschen Bauernverbands, in: Festschrift für A. Hermes, Neuwied 1958, S. 181-196

Jürgensen, Kurt, Schleswig-Holstein - das „Modell-Land“ der britischen Besatzungspolitik, in: Studien zur europäischen Geschichte. Gedenkschrift für M. Göhring, Wiesbaden 1968, S. 396-412

Kaack, Heino, Geschichte und Struktur des deutschen Parteiensystems, Opladen 1971

Kaden, Albrecht, Einheit oder Freiheit. Die Wiedergründung der SPD 1945/46, Hannover 1964

Kluth, Hans, Die KPD in der Bundesrepublik. Ihre politische Tätigkeit und Organisation 1945-1956, Köln 1959

Kotow, G. G., Agrarverhältnisse und Bodenreform in Deutschland, Teil 2, Berlin (Ost) 1959

Lange, Max, Gerhard Schulz, Klaus Schütz u. a., Parteien in der Bundesrepublik. Studien zur Entwicklung der deutschen Parteien bis zur Bundestagswahl 1953, Stuttgart 1955 
Leitsätze der Gesellschaft zur Förderung der inneren Kolonisation zur Durchführung der Bodenreform, o. O. 1947

Lübke, Heinrich, Bodenreform - Siedlung - Erzeugungssteigerung, Vortrag auf der Tagung der GFK am 30.10.1947, Lüneburg 1948

ders., Grundlagen einer neuen Siedlungspolitik, Recklinghausen 1946

Meyn, Hermann, Die Deutsche Partei. Entwicklung und Problematik einer nationalkonservativen Rechtspartei nach 1945, Düsseldorf 1965

Monthly Report of the Control Commission for Germany (BE), Berlin 1946-1950

Niedersächsische Volksstimme [Organ der KPD], Hannover 1946-1949

Niedersächsischer Landtag, Stenographische Berichte der 1.-9. Sitzung (Ernennungsperiode), Hannover 1946/47, der 1.-68. Sitzung (1. Wahlperiode), Hannover $1947 \mathrm{ff}$.

Niedersächsisches Gesetz- und Verordnungsblatt 1947-1954, Hannover $1947 \mathrm{ff}$.

Niehaus, Heinrich, Theorien, Vorschläge und Gesetze zur Agrarreform, in: Hefte für Agrarwissenschaft und Agrarpolitik 1 (1947)

Nordrhein-Westfälischer Landtag, Stenographische Berichte der 28.-76. Sitzung (1. Wahlperiode), Düsseldorf 1948/49

Pakenham, Lord F., Die britische Aufgabe in Deutschland, in: Neues Europa 2 (1947)

Passarge, Karl (Hrsg.), ZEL. Zentralamt für Ernährung und Landwirtschaft in der britischen Zone 1945-1948. Ein Rückblick, Hamburg 1948

Pieck, Wilhelm/Hoernle, Edwin, Demokratische Bodenreform, Berlin 1945

dies., Bodenreform. Junkerland in Bauernhand, Dresden 1945

Pirker, Theo, Die SPD nach Hitler. Die Geschichte der SPD 1945-1964, München 1965

Protokoll der Gewerkschafts-Konferenz der britischen Zone vom 21.-23. 8. 1946 in Bielefeld, hrsg. vom Gewerkschaftlichen Zonensekretariat, Bielefeld 1947

Protokoll des Gründungs-Kongresses der DGB vom 22.-25. 4. 1947 in Bielefeld, hrsg. vom DGB-Bundesvorstand, Düsseldorf 1947

Pünder, Hermann, Von Preußen nach Europa. Lebenserinnerungen, Stuttgart 1968

Pünder, Tilman, Das bizonale Interregnum. Die Geschichte des Vereinigten Wirtschaftsgebietes 1946-1949, Köln 1966

Reuber, Heinrich/Skibbe, Bruno (Hrsg.), Die Bodenreform in Deutschland mit Zahlen und Dokumenten, Berlin 1947

Ritter, Waldemar, Kurt Schumacher. Eine Untersuchung seiner politischen Konzeption und seiner Gesellschafts- und Staatsauffassung, Hannover 1964.

Röder, Werner, Die deutschen sozialistischen Exilgruppen in Großbritannien 1940 bis 194.5, Hannover 1968

Rudzio, Wolfgang, Die Neuordnung des Kommunalwesens in der britischen Zone. Zur Demokratisierung und Dezentralisierung der politischen Struktur. Eine britische Reform und ihr Ausgang, Stuttgart 1968

Sammlung zum Aufstieg. Eine zusammenfassende Darstellung der Beratungen und Beschlüsse des Parteitages 1948 der SPD vom 11.-14.9. in Düsseldorf, Hannover 1949

Schlange-Schöningen, Hans, Justus Rohrbach, Im Schatten des Hungers. Dokumentarisches zur Ernährungspolitik und Ernährungswirtschaft in den Jahren 1945 bis 1949, Hamburg 1955

Schleswig-Holsteinischer Landtag, Wortprotokolle der 1.-9. Sitzung des 1. (ernannten) Landtags, Kiel 1946, der 1.-7. Sitzung des 2. (ernannten) Landtags, Kiel 1946/47, der 1.-35. Sitzung des 3. (ersten gewählten) Landtags, Kiel 1947-1950

Schmalz, Hellmuth, Als Adam grub und Eva spann ... Gedanken zur Bodenreform, Hannover o. J. (1947)

Schmidt, Eberhard, Die verhinderte Neuordnung 1945-1952. Zur Auseinandersetzung 
um die Demokratisierung der Wirtschaft in den westlichen Besatzungszonen und in der Bundesrepublik Deutschland, Frankfurt 1970

Schoenberg, Hans W., Germans from the East. A study of their migration, resettlement and subsequent group history since 1945, The Hague 1970

Schumacher, Kurt, Bundestagsreden, hrsg. von Annemarie Renger, Bonn 1972

ders., Reden und Schriften, Berlin 1962

ders., Nach dem Zusammenbruch. Gedanken über Demokratie und Sozialismus, Hamburg 1948

Schwarz, Hans-Peter, Vom Reich zur Bundesrepublik. Deutschland im Widerstreit der außenpolitischen Konzeptionen in den Jahren der Besatzungsherrschaft 1945 bis 1949, Neuwied/Berlin 1966

SOPADE. Informationsdienst. Hrsg. von der SPD, Hannover $1946 \mathrm{ff}$.

SOPADE. Querschnitt durch Politik und Wirtschaft. Hrsg. von der SPD, Hannover $1947 / 1948$

SPD. Wochenschrift für Sozialismus und Demokratie, Hannover $1946 \mathrm{ff}$.

SPD, Bezirk Hannover (Hrsg.), Der Kampf um die Bodenreform in Niedersachsen, Hannover 1948

Stamer, Hans, Die wirtschaftlichen Auswirkungen der ländlichen Siedlung, Kiel 1955

Stammen, Theo, Einigkeit und Recht und Freiheit. Westdeutsche Innenpolitik 1945 bis 1955, München 1965

Start zur Bewährung. Eine zusammenfassende Darstellung der Beratungen und Beschlüsse des Parteitages 1947 der SPD vom 29. 6.-2. 7. in Nürnberg, Hannover 1947

Steltzer, Theodor, Sechzig Jahre Zeitgenosse, München 1966

Treue, Wilhelm, Die wirtschaftsgeschichtliche Entwicklung Niedersachsens seit 1764, in: Die Landwirtschaft Niedersachsens 1914-1964, Hannover 1964, S. 57-107

Treue, Wolfgang, Deutsche Parteiprogramme 1861-1961, Göttingen 1961

Ulbricht, Walter, Die demokratische Bodenreform - ein ruhmreiches Blatt in der deutschen Geschichte, in: Einheit 10 (1955), S. $84.9 \mathrm{ff}$.

Vogel, Walter, Westdeutschland 1945-1950. Der Aufbau von Verfassungs- und Verwaltungseinrichtungen über den Ländern der drei westlichen Besatzungszonen, Teil 1, Koblenz 1956

Vogelsang, Thilo, Einführung in die Problematik. Westdeutschland zwischen 1945 und 1949. Faktoren, Entwicklungen, Entscheidungen, in: VfZG 21 (1973), S. 166 bis 170

ders., Das geteilte Deutschland, München $1973^{5}$

ders., Hinrich Wilhelm Kopf und Niedersachsen, Hannover 1963

Wagner, Bruno, Die Bodenreformpolitik der SPD, Berlin 1958

Weisz, Christoph, Organisation und Ideologie der Landwirtschaft 1945-1949, in: VfZG 21 (1973), S. 192-199

Wieck, Hans-Georg, Die Entstehung der CDU und die Wiederbegründung des Zentrums 1945, Düsseldorf 1953

Wirtschaftsrat des VWG, Wörtliche Berichte über die Vollversammlungen des Wirtschaftsrates des VWG. 1.-40. Sitzung, Frankfurt 1947-1949

Zeitschrift für das gesamte Siedlungswesen, hrsg. von der Gesellschaft zur Förderung der inneren Kolonisation, Düsseldorf $1952 \mathrm{ff}$. 


\section{Personen- und Institutionenregister}

Abel, Karl (KPD) 136

Adenauer, Konrad (CDU) 24, 38-40, 65, 119

Agartz, Viktor (SPD) 35

Agrarsoziale Arbeitsgruppe (Göttingen) 96

Albers, Johann (DP) 135

Allen (Colonel) 52

Amelunxen, Rudolf (Zentrum) 65

amerikanische Zone 15-17, 31, 54, 59, 73, 80, 86 f., 94, 104, 106 f., 111, 163, 165 f. Arndt, Gerhard (CDU) 144.

Arnold, Karl (CDU) 70, 119, 133

Arbeitsgemeinschaft für Agrarfragen (Hannover) 91, 95, 149, 161

Arbeitsgemeinschaft der deutschen Bauernverbände $89,92,105,162$

Arbeitsgemeinschaft des Grundbesitzes (Kiel) 118, 127, 129

Arp, Erich (SPD) 64, 84, 109, 112, 114, 126

Bevin, Ernest 53, 60, $73 \mathrm{f}$.

Bidault, Georges 18, 74.

Bipartite Control Office (BICO) 106, 126

Bishop, William Henry Alexander $75 \mathrm{f}$, $102,133 \mathrm{f}$.

Bizone 11, 20, 53, 75, 96, 106, 169

Block, August (NLP/DP) 47, 66, 73, 77, 87, 109, $123 \mathrm{f} ., 135-138,140,148 \mathrm{f}$., 153, 155

Bode, Wolfgang (NLP) $80 \mathrm{f}$.

Bödeker 30

Boyens, Wilhelm Friedrich 63

Bresk 47, 96

Britische Militärregierung (BIMR) 22-25, 27-29, 31, 41, 48f., 55, 57, 60, 66, 75, $79,81-85,89,92,101 \mathrm{f} ., 107 \mathrm{f} ., 114$ bis $116,118,120,122,130,132-134,140$, 152 f., 155, 157-159, 161, 167

britische Regierung 19, 26, 53, 57, 76, 78, 93, 115, 166

Brockdorff-Ahlefeldt, Graf v. (CDU) 27

Broederich, Silvio (GFK) 30, 48, 94 .

Brüning, Heinrich 120

Byrnes, James F. 53
CDU 14, 18, 23, 29, 38-40, 42, 64 f., 67, 70-72, 80, 82, 86, 88 f., 91 f., 94, 99, 103-105, 110, 112, 117 f., 127-133, 141, 143, 145-147, 151-155, 161, 166-172 Cillien, Adolf (CDU) 71, 145

Clay, Lucius D. 11, 15-17, 165

Deutscher Bauernverband (DBV) 91-95, 99, 103 f., 153, 166, 172

Deutscher Gewerkschaftsbund (DGB) 25, 29, 47, 54 f., 75, 96 f., 99, 127

Deutschland-Ministerium, brit. 61, 100

Diederichs, Georg (SPD) $139 \mathrm{f}$.

Diekmann, Bruno (SPD) 128

Douglas, Sir Sholto 24

European Advisory Commission 9

Everling, Henry 30, 47

FDP 29, 42f., 65, 80, 89-92, 103-105, 119, 131, 142, 145, 147, 152, 155, 172

Ficker, Ludwig (KPD) 105

Food \& Agriculture Division, brit. 55, 60

französische Zone 17 f., 74, 163

Gereke, Günther (CDU) $141 \mathrm{f} ., \quad 152$, 154 f., 157 f., 160 f.

Gesellschaft zur Förderung der inneren Kolonisation (GFK) $29 \mathrm{f}$., 47-49, 91, 94f., 119, 161

G I F A C 23

Gülich, Wilhelm (SPD) 117, 128

Hannover, Provinz 29, 44, 65

Haxsen, Ottomar (NLP/DP) 45

Heile, Wilhelm (FDP) 24, $42 \mathrm{f}$.

Hellwege, Heinrich (DP/NLP) 44-46, 73, $88,102,129,135,145,153 \mathrm{f}$.

Henssler, Fritz (SPD) 29, 31, 37, 69, 75, 81,84

Hermes, Andreas (CDU) 14, 92, 166

Hoffmann, Erich (KPD) 33, 82

Hofmeister, Robert (SPD) 146

Hollins, Frank 23, 41, 48 f.

Hummel, Johannes (CDU) 92, 153

Hynd, John B. 52, 61 
Jürgensen, Julius (KPD) 33, 82

Kaiser, Jakob (CDU) 38

Karl, Albin 81

Klatt, Werner $60 \mathrm{f}$., 100, 106

Knigge, Freiherr v. 95

Knoop, Hugo (FDP) 30, 42, $80 \mathrm{f}$.

Konferenz der Ministerpräsidenten in der britischen Zone (KdM) 26, 111-115

Kontrollkommission, brit. 26-29, 31, 55, 61 f., 76, 82 f., 88, 90, 100, 165

Kontrollrat, Alliierter 10, 12, 14-17, 19, 21, 26, 52, $72 \mathrm{f}$., 91

Kopf, Hinrich Wilhelm (SPD) 24, 29, 44, 65-67, 113-115, 123 f., 135, 142-146, $151-154,157-160$

K PD 13 f., 29 f., 32-34, 37, 45, 51, 54, 66 f., 72, 80-84, 97, 102 f., 131 f., 135, $137,141,144-146,149,152,158 \mathrm{f}$.

Krapp, Otto (Zentrum) 142

Kriedemann, Herbert (SPD) 29, 34-37, 68 f., 79-82, 85, 87, 102, 105

Labour Party 60 f., 72

Länderrat (US-Zone) 16, 111

Lauenstein (NLP/DP)

L D P D 14

Lingham, John 144, 153 f., 157 f., 160

Lippe 29

Lübke, Friedrich Wilhelm (CDU) 117

Lübke, Heinrich (CDU) 39, 41, 48, 70, 75, 82, 87, 94, 109, 113f., 119-121, 127, $130,132-134$.

Lüdemann, Hermann (SPD) 113

Luster-Haggeney, Franz (CDU) 92

Machui, Artur v. 49, 68, $96 \mathrm{f}$.

MacReady, Gordon 66, 167

Marreco, Anthony 165

Meyer-Bülkau, Heinrich (NLP/DP) 47, 66,140

Molotow, Wjatscheslaw Michailowitsch 13,73

Morgen 30

Morgenthau, Henry 15, 35

Müller, Karl (CDU) 29, 41 f., 80 f., 87 f.

Müller, Kurt (KPD) 82

NL P/DP 29 f., 44-47, 66 f., 71-73, 80, 82, 87-92, 94f., 102-104, 110, 122, $124,129,135,140 \mathrm{f}$., 143-149, 151 bis $153,155,161,172$
Niedersächsisches Landvolk (NLV) $91 \mathrm{f}$, $94,103,149,156,161$

Niehaus, Heinrich $92 \mathrm{f}$.

Nonhoff (ZEL) 59

Nordrhein-Westfalen 41, 65, 67, 70, 107 f., 112 f., 116, 119 f., 125 f., 129 f., $134,151,157,163-165,167-172$

\section{Oldenburg 29}

OMGUS 16, 21, 154, 165

Otto, Paul (CDU) $41 \mathrm{f}$.

Palmer, Siegfried 126

Paul, Hugo (KPD) 81-83

Podeyn, Hans (SPD) 49

Potsdamer Konferenz (Abkommen) 10, 12, 15 f., 19 f., 23, 26, 52, 54-56

Reden, Baron v. 113

Rehwinkel, Edmund (NLV) 92, 156

Reichs-Landbund 35, 68, 93

Reichsnährstands-Verwaltung 22, 54

Reimann, Max (KPD) 24, $80 \mathrm{f}$.

Robertson, Sir Brian Hubert 23, 26 f, 74 f., 80 f., 100, 115, 157, 165, 167

Schleswig-Holstein 20, $22 \mathrm{f}$., 29, 60, 63 f., $70,72,84,95,107$ f., 112-115, 116-119, 125-129, 163, 165 f., 169

Schlange-Schöningen, Hans (CDU) 21, 23 f., 27, 29, 38-41, 44, 48f., 51, 54f., $60 \mathrm{f} ., 63,70,75,81,95-97,106 \mathrm{f}$., 109 f., 126

Schmalz, Hellmut (KPD) 67, 139, 141, 146,160

Schreiber, Walther (CDU) 14

Schröder, Bruno (FDP) 142

Schröter, Karl (CDU) 81, 87, 99, 117, 127

Schulenburg-Lüerdissen, Graf v. d. 95

Schumacher, Kurt (SPD) 24, 34-36

Seebohm, Hans-Christoph (NLP/DP) 135

Siemer, Hermann-Josef (CDU) 81, 139, 152

Sonderausschuß des Zonenbeirats für Agrarreform (1. SAfAR) 28-34, 40-42, $46,48,50,61 \mathrm{f}$.

Sonderausschuß des Zonenbeirats für Agrarreform (2. SAfAR) 78, 81-83, $87 \mathrm{f} ., 90 \mathrm{f}$.

Sowjetische Besatzungszone 8, 10, 12-15, $17,25-27,29,32-36,38,40-43,46,49$, 51,67 f., $97,117,170-172$ 
S P D 14, 18, 24, 29, 34-38, 41, 45-47, 54f., 64, 67-72, 79f., 84-87, 89, 92 f., 99, 102 f., 105, 107, 112, 116-119, 125 bis 127, 130-132, 135-139, 141-146, 149-155, 158, $171 \mathrm{f}$.

SPD-Agrarpolit. Ausschuß (ApA) 68f., $84,102,130,150$

Spiecker, Carl (Zentrum) 80

Steffen, Friedrich (NLP/DP) 47

Steltzer, Theodor (CDU) 24, 29, 40 f., 63, 70

Stricker, Fritz (Zentrum) 81

Tantzen, Theodor (FDP) $29 \mathrm{f} ., 42$

Teichmann, Ulrich (DGB) $96 \mathrm{f}$.

Truman, Harry S. 73

U N O 23,52

Unterhaus, brit. 52,54

US-Militärregierung 12, 15

Verbraucherverbände (Konsumgenossenschaften) $25,29,36,43,47,88$
Vereinigtes Wirtschaftsgebiet (VWG) 10, 53,104

VE L F $17,53,87,106-112$

Weisser, Gerhard (SPD) 29

Wirtschaftsrat des VWG 75, 78, 87, 91, 94, 104-106, 110, 126, 128, 165 f.

Wirtschaftsrat (Ernährungsausschuß) 62

Zentralämter 19 f., 25, 41, 47

Zentralamt für Ernährung und Landwirtschaft (ZEL) 21, 23 f., 27, 29-31, $33,39,41,46,48-50,53,55,59-62,64$, $76 \mathrm{f} ., 79,81$ f., $92,100,106,108$

Zentrum 80, 89, 91, 121, 131, 142 f., 145, 152

Zonenbeirat 20, 24ff., 27-32, 34, 36, 40 bis $42,44,48,50,52-55,58 \mathrm{f} ., 61 \mathrm{f} ., 64$, 66-70, 74-79, 81-84, 86-89, 90, 92, 94, $96,100-102,104,119,146$

Zweizonenamt f. Ernährung, Landwirtschaft und Forsten (ZZVELF) 108, 111 